\section{解nterian Pertures}

on

\section{MAN'S POSTURE: ITS EVOLUTION AND DISORDERS.}

Given at the Royal College of Surgeons of England

BY

Profegsor Sir ARThUR KEITH, F.R.d.S., F.R.S., CONSERVATOR OF THE MUSEOM.

\section{[Abstracts.]}

\section{LRCTURE V.-POSTURAL MODIFICATIONS OF THE} PELVIS, PERINEUM, AND GROIN.

NATORE in her evolutionary creations has often, like modern statesmen, to effect a compromise between contending interests. I may cite the human pelvis as an instance to illustrate my meaning. The pelvis has a locomotory interest : it is an extremely complex lever by which the muscles of the hip and buttock balance the weight of the trunk on one or on both lower limbs. Then it has a visceral interest; it has not only to contain certain organs, but must also give suitable attachment to the musculature which supports the contents of the abdomen. It has a spinal interest in that it affords the chief base for the musculature of the vertebral column. It has an obstetrical interest, having to be so framed in women as to give a safe passage to the child at birth. It has to give attachments to the external genital organs and to the terminal parts of the rectum and urethra. Even the most hypercritical of anatomists and surgeons agree that Nature has combined these contending functional interests in the pelvis of man with the utmost ingenuity. No one has been able to suggest an improvement on her design, and yet the fact remains that in modern civilized communities pelvic defects, among which I would classify hernia and prolapse, are very common.

\section{Are Hernia and Prolapse to be Reckoned as Postural} Defects?

Are we to say, then, that Europeans and peoples of European origin are more liable to hernia and to uterine prolapse than more primitive peoples, such as the aborigines of Australia, because the European body is less perfectly adapted to the upright posture? I think not; the Australian aborigine spends his days amidst conditions very similar to those for which early man was evolved, while our conditions of living and working are of recent development, and very different from the aboriginal kind. We shall be wise to search in these modern conditions of life for the factors which contribute to our liability to hernia rather than to lay the whole blame on postural imperfections of our bodies. Two of these modern factors solicit the attention of medical men. The first of these is the states of high pressure which arise within the abdominal cavity of modern labourers during violent effort, as in lifting heavy weights, or in the physically weak during fits of coughing or of straining at stool. On standing up the pressure on the floor of the pelvis, taken from within the passive rectum and registered on a mercury manometer, will be found to vary from 15 to $25 \mathrm{~mm}$. so long as we stand still, but the moment we attempt any arm movement, particularly if we bend down and seek to lift a heavy weight, the intra. polvic pressure, as registered on the manometer, instantly rises to the neighbourhood of 80 to $100 \mathrm{~mm}$. If the effort is made suddenly the initial rise is much greater-reaching as much as $150 \mathrm{~mm}$. In all such movements the visceral con. tents of the abdomen are compressed within the envelope of the postural musculature and seek out the weakest points in the containing walls: they act as water-hammers. No one will deny that modern civilization has increased the abdominal strain for labouring men and women.

Probable Effects of Errors in Diet.

Then there is another modern factor at work, to which attention has been already drawn. In modern communities visceroptosis is common; we cannot explain this postural displacement of the abdominal viscera except by supposing that the reflex mechanism which controls the supporting musculature has become damaged. Such damage to the musculature of the abdominal and pelvic walls must increase the liability to pelvic prolapse and to hernia. But when we have made all allowances for these two factors-increased intra-abdominal stresses and disordered action of the parietal musculature-the fact still remains that the modification of man's pelvis to answer the needs of plantigrade progression has wealzened the pelvic walls, particularly in the regions of the groin and of the pelvic outlet.

Defence of the Pelvic Outlet.

The defensive structures of the outlet of the human pelvis are represented by the levator ani muscles and certain fascial and ligamentous structures. The human type of pelvic floor is not peculiar to man; it is found in all members of the orthograde group of primates-in the great anthropoids, in the gibbons or small anthropoids as well as in man. It may be called the orthograde type of pelvic floor and was evolved with the appearance of the orthograde posture. Reasons have already been given for deriving the earliest orthograde forms from a pronograde ancestry. Now in all pronograde apes, whether the tail be long or short, its root is made to serre the purpose of a perineal shutter. It was, as we shall explain, the evolution of the orthograde pelvic floor which led to the coccygealization of the tail. An external tail had dis. appeared from the orthograde stock long before the human form came into being.

\section{The Pronograde Defence.}

We can best realize the extent to which the defensive mechanism of the pelvic outlet underwent a postural transformation if we compare the pelvic structures of a pronograde ape, such as a macaque, with the same parts in the ortho. grade gibbon. However short the tail may be, it will be found to have its own proper muscles-muscles which rise from dorsal and ventral aspects of the sacrum and have the power to extend, flex, and move the tail from side to side. On the ventral or perineal aspect of the second, third, and fourth vertebrae of the tail are small V-shaped or cherron bones to which the guardian muscles of the pelvic outlet are attached. The guardian muscles of the outlet-which become modified to form the levator ani muscles of the orthograde pelvis-are arranged in two pairs. The median pair-the pubo-caudal muscles-arise within the whole length of the symplyysis pubis, and, leaving between them a narrow chink through which escape the urethra, vagina, and rectum, run backwards to the chevron bones beneath the base of the tail. The lateral pair-the ilio-caudal muscles-arise from the brim of the pelvis and pass to the cherron bones of the tail. These muscles have fascial sheaths, so have the pelvic viscera; muscular and visceral sheaths fase at the chink of exit. The arteries and nerves passing to the pelvic viscera are clothed with connective tissue sheathing to guard them from being strained when the floor y:elds. Thus the prono. grade pelvic outlet is guarded by muscles to which the pelvio viscera have fascial attachments.

In the horizontal gait the pelvic outlet is the highest point of the abdominal cavity; when a human patient is placed in a similar position-on elbows and knees-the pelvic pressure becomes negative. But if the patient strains or attempts to rise the intrapelvic pressure at once mounts up. It is so in the active pronograde monkey. When preparing to jump it will be observed that the muscles which guard the outlet of the pronograde pelvis immediately depress the root of the tail and close the outlet. In defaecation the tail is raised, the median intermuscular chink is lengthened; the pubo-caudal or guardian muscles then act between two fixed points-the pubis and base of the tail.

\section{Shedding of the Tail.}

Conceive for a moment what would happen were a prono. grade monkey to assume the orthograde posture of a gibbon. The lower end of the column, formed by the visceral contents of the abdomen, would then come to rest on the guardian muscles of the pelvic outlet-the levatores ani. The muscles would then contract and close the pelvic outlet by depressing the root of the tail; so long as the animal maintained the orthograde posture the muscles would have to keep con. tracted and the tail depressed and immobile. The pubocaudal and ilio-caudal muscles have thus become postural in function when an upright position is assumed, and the depressed tail has become, except for its basal part, a useless structure. Now when a structure becomes useless it disappears; we do not know the process which Nature uses to get rid of useless structures, but we do know that the tail of the tadpole withers when the aninal passes into the frog stage, and that the shedding of the tail is dependent on a 
full action of the thyroid gland. We are certain, at least, that with the first stage in the evolution of the orthograde posture the external tail disappeared; the basal vertebrae became vestigial or coccygealized. The coccyx of the gibbon is more reduced than even that of man; it has reached its most atrophic form in the orang.

The Orthograde Pelvio Floor.

With the coccygealization of the tail the pubo-caudal and vilo-caudal muscles of the pronograde pelvis became spread out to form a supporting muscular hammock, but still attached to the vestige of the tail. Man's pelvic floor is an ancient heritage; it came into being with the evolution of the oldest orthograde type.* Not only have the attachments and arrangement of the pelvic muscles been modified to suit the assumption of a new posture, but the nerve mechanisms which control these muscles must also have become modified.

Evidence of the Former Existence of a Tail.

What evidence have we that the tailless pelvic floor of man and of the orthograde apes was evolved from one which was tailed and pronograde in type? Our evidence is most complete as regards man. Children are occasionally born with true tails; in the sixth week of development the human embryo has a free projecting tail, containing nine to twelve segments; by the twelfth week the terminal three or four vertebrae have been absorbed, and a dimple on the rump marks the point at which the free tail has sunk below the surface. Most significant of all is the presence in the tail of the human embryo of vestiges of the chevron bones, which give attachment to the muscles of the pelvic outlet in prono. grade monkeys. We cannot explain the vestiges of chevron bones in our bodies, nor of a caudal lobe in the liver, nor of the azygos lobe of the right lung, unless we suppose that there was a pronograde stage in man's ancestry.

\section{Evolution of the Human Perineum.}

The outlet of the human pelvis is a diamond-shaped space, made up of rectal and urethral triangles. The posterior, rectal, or ischial part of the outlet is old; the anterior, urethral, or pubic part of the space is new. In pronograde monkeys the ischial tuberosities come almost into contact; the whole outlet in them represents only the posterior part of the outlet of the human pelvis; only a rudiment of the pubic part of the space is present. In the gibbon the ischial tuberosities and rami are as in pronograde apes, but in the orang, chimpanzee, and more especially in the gorilla, the ischial tuberosities do not meet and there is the beginning of the subpubic space which becomes so marked a feature of the human pelvis, particularly the pelves of European women. In anthropoids the perineum is placed on the dorsal surface of the body; the roomy outlet of the pelvis looks backwards; the symphysis forms the lowest part of the pelvis. The levatores ani enter into the formation of the posterior wall of the pelvis rather than form a floor for it. In the hnman pelvis the posterior part of the outlet has been diminished in size and strengthened in its frame. work (1) by a bending forwards of the apical part of the sacrum and a strengthening of the vertebrae of the coccyx (2) by a strengthening of the great sacro-sciatic and small sacro-sciatic ligaments. These ligaments are present only in orthograde primates. But if the human pelvic outlet has been strengthened posteriorly it has been weakened anteriorly by the opening out of the subpubic arch. Also the symphyseal part of the human pelvis has been tilted upwards, in the direction of the umbilicus; it comes to occupy a higher level, taking the coccyx as a guide or mark, than is the case in man's nearest allies, the great anthropoid apes. These changes have brought the human vulval orifice towards the anterior or ventral surface of the body. They have also brought the levator muscles into a more borizontal position so that in man they form a pelvic floor designed to support the abdominal contents.

Pelvic Supports Strongest in Man.

At this point I cannot forbear giving a quotation from the writings of John Hunter." "The contents of the luman pelvis," he wrote, "adhere to the sides by a much greater extent of surface than in any other animal. The intention of this is perhaps to prevent a protrusion of these parts (the

* For fuller details see the late Professor Peter Thompson's Myology of The Pelvic Floor, Manchester, 1899; Dr. R. H. Paramore's Hunterian Morphology, fourth edition, 1921. womb, the bladder, the rectum, and bowel) from the weight of the viscera above, in the erect posture of man." Since the above passage was written, some one hundred and fifty years ago, we have learned that these pelvic adhesions are almost equally extensive in the orthograde primates. All have wide adhesions, fascial unions, and vascular supports, but in strength and toughness they reach their climax in man. These adhesive supports came with the evolution of the orthograde posture. In all pronograde forms only part of the bladder and uterus lie within the pelvis. In the human infant, as in the young of anthropoids, the pelvic viscera are situated as in pronograde apes; but in the adults of anthro. poid, as of man, bladder, uterus, and ovaries have sunk within the cavity of the pelvis. A natural visceroptosis takes place at the lower end of the abdominal cavity of all orthograde primates as adolescence is reached.

The Dimensions of the True Pelvis.

We are apt to think of the human pelvis as being excep. tionally capacious. The conjugate diameter of the pelvis of the gorilla is often double that of the human pelvis; in the chimpanzee the conjugate is half as much again as the human. In transverse width the true pelvis of the gorilla attains human dimensions, while in the chimpanzee it falls short. In depth the anthropoid pelvis is the greater and its outlet the more roomy. In them there is no pelvic bar to the birth of big-headed progeny. And yet, in spite of their orthograde posture, they do not seem to suffer from the forms of uterine prolapse to which women are so liable.

The Weak Point of the Female Perineum.

Why, then, are women, particularly women of European stocks, so liable to uterine prolapse? The weakest point in woman's pelvic floor is represented by the vaginal passage; the vaginal canal, like the inguinal and femoral canals, may become the site of a hernia. It is not the mere presence of the vaginal passage which makes a woman liable to pro. lapse; the rectal or anal passage would be equally weak, did it not possess strong muscular coats guarded by efficient sphincters. The vagina has neither strong muscular coats nor an efficient sphincter. In the anthropoids this passage is no better protected than is the human one, yet it does not become the site of prolapse. No doubt the opening up of the subpubic space and the tilting up of the pubic part of the pelvis to bring the vulva towards the ventral surface of a woman's body has weakened the vaginal and vesical areas of her pelvic floor. But the chief causes of woman's liability to prolapse have to be sought for, as is the case in all hernial formations, in the high and varying degrees of pressure generated within the abdominal cavity in consequence of the plantigrade posture. As already pointed out, when we sit or stand the column of abdominal viscera becomes the core within a compressing cylinder of postural musculature. Every movement of the arms, every cough or strain, sets going a multitude of water. hammers within the abdominal and pelvic cavities which search out the weak points in the surrounding walls. Just over the vaginal passage is the fluid-containing bladder; every intra-abdominal impulse sets the bladder knocking at the vaginal exit. At the upper end of the vagina is set the cervix of the uterus; it too responds to intra-abdominal pressures and strains, beating downwards into the passage. It is the continual repetition of small forces, more frequently than the sudden application of a great effort, which wears down the raginal defence.

In cases of uterine prolapse, several of which I have had an opportunity of examining after death, I have found the muscles of the pelvic floor elongated but not atrophied. The ligamentous supports had become stretched; the pelvic structures had been forced outwards. Yet, I think, it will be found that prolapse would not have taken place if the musculature of the pelvic floor and the musculature of the vagina and supporting tissues of the uterus had been normal in tone and possessed the reflex reactions of health. There is a great lack of any real evidence on this point, but of this there can be no doubt-the idle and luxurious are just as liable to these infirmities as are women who have to perform heavy labour.

The Evolution of the Human Groin.

We now turn to the human groin to see if our knowledge of its evolution can throw any light on its two weal pointsthe inguinal canal and femoral ring. In all primates, in 
consequence of an elaborate series of developmental or growth rrocesses, parts of the abdominal cavity containing the testes are extruded and come to occupy a position in the scrotum. In pronograde monkeys the peritoncal passage, although very narrow, remains open; in orthograde primates it usually becomes closed; but even in man, in whom the process of closure is most complete, the canal remains unclosed in 30 per cent. of newly born children. In all primates, then, there is a breach in the musculature of the groin caused by the descent of the testes or of the round ligament. In all there is a second breach in the groin caused by the passage of the great blood vessels from the abdominal cavity to the thigh. The vascular aperture of the groin must vary in size; at times the vessels-particularly the vein-are grcatly distended; at other times they are partially collapsed, thus giving a potential space for the escape of abdominal contents. In man the femoral ressels are of exceptional dimensions in consequence of the massive development of his thighs; hence in him the vascular apertures of his groin are large. It is not the presence of an inguinal canal or femoral ring which makes the human groin exceptionally liable to become the site of hernia, for these apertures are a common possession of the whole order of primates.

\section{The Groin of Apes.}

The human groin is one of the most distinctive features of man's anatomy; it is in a class by itself ; in only one animal do we find a tendency towards the human form, and that is in the gorilla. If we did not possess some of the intermediate stages it would be difficult to believe that the human groin had been evolved from a pronograde form, such as is seen in the commoner monkeys. In monkeys the infraumbilical part of the belly has a narrow keel-like form terminating posteriorly at the sharp projecting symphysis pubis; on each side is the long narrow furrow or fold of the groin, separating the abdomen from the flat inner surface of the thigh. The two groin furrows form a V-shaped arrangement, the limbs of the $\checkmark$ ending above at the anterior superior iliac spines, which are buried in the flanks. In the fold of the monkey's groin can be felt the particularly long anterior edge of the ilium, offering a firm line of attachment for the musculature which controls the apertures of the groin-the lower parts of the internal oblique and transversalis muscles. The groin is further strengthened by the fact that the external oblique muscle has no insertion to the iliac crest, but expends its strength on rendering tense the structures round the apertures of the groin. The whole arrangement makes up a most competent sphincteric mechanism; although the peritoneal passage to the scrotum remains open, escape of the abdominal contents is very rare. Further, these guardian muscles are under a reflex control.

The Orthograde Groin.

With the evolution of the simian orthograde posture the form and structure of the groin underwent surprisingly little change. In the gorilla we find a premonition of the changes which have transformed the human groin. The first of these is the extension of the crest of the ilium in an outward and also in a slightly forward direction. The fibres of the external oblique muscle, which rise from the lower ribs, no longer sweep to the groin but are inserted to the anterior end of the extended iliac crest; there is also the first appearance of the inguinal (Poupart's) ligament, formed out of the tendinous fibres of that part of the external oblique which is inserted to the crest. Further, in the gorilla the anterior border of the ilium, lying along the fold of the groin, has become relatively short. In man these changes liave reached a climax; the crest of the ilium has become extensive and has undergone a marked bend forwards; the external oblique has gained a powerful insertion to the crest, and Poupart's ligament. has become a strong and differentiated fibrous bridge between the anterior superior iliac spine and pubic spine. Lastly, the anterior border of the ilium has been reduced to a little more than a third of the primitive length seen in great anthropoids; the long iliac border which gave so secure an origin for the guardian muscles of the groin has gone. The lower parts of the internal oblique and transversalis muscles. have now to seek a lests advantageous origin from a new basenamely, Poupart's ligament. The folds of the liuman groin hare been shortened and opened out, and thus assumed a wide U-shaped form. The lower abdominal region lias become Haltened in front.
All these changes have weakened the defensive mechanism of the human groin. To understand the meaning of these changes one has to remember that the pelvis is made up of a series of levers, which serve in balancing the body on the thigh. The climbing habits of the great anthropoids demand long and powerful pelvic levers; to suit man's plantigrade needs, to balance the body quickly and easily as he walks, short pelvic levers with sliort-fibred but powerful gluteal muscles become necessary; hence the transformation of his pelvis and shortening of his groin. It is not the orthograde posture which weakened the human groin but the transformation of his pelvis to suit the needs of plantigrade progression. As we have already seen, plantigrade progression is neces. sarily attended by high degrees of intra-abdominal tension. Modern methods of living-exactly how we do not knowcan damage the reflex postural mechanism of the belly wall. Man's liability to hernia must be sought for in all these factors.

The Liability to Femoral Hernia.

At the beginning of this lecture I pointed out that the human pelvis was the sport of contending interests. In answering the needs of locomotion it has become less efficient as a part of the abdominal wall. In the liability of women to femoral hernia we see a disharmony between the sexual and containing functions of the pelvis. At puberty a growth takes place in the pelvis of women at three sites-at the symphysis pubis and at the two ilio-sacral joints. The new bone laid down at these joints leads to an increase of all diameters of the pelvis. Exactly the same kind of growth is seen in the pelves of men siffering from the later stages of acromegaly; the sexual transformation of the pelvis is the result of a growth mechanism contro.led by hormones. In women, in whom the sexual growth is malked, the pabic part of the pelvis becomes elongated and the vascular compartment under Pourart's ligamen't widened. ${ }^{2}$ Hence an increased liability to femoral hernia in women. A femoral sac is never the rcsult of a developmental process; it is a structure which has never been seen at any stage in the development of luman embryos or foetuses.

\section{REFERENCES.}

1 Essays and Observations, edited by Sir Richard Owen, vol. i, p. 45 Secent parer on this subject by Dr. J. Allison Panton, Journ 2 See a recent parer on
Anat., 1923, vol. 57, p. 106.

\section{VENOUS PULSATIONS AND VENOUS TRACINGS IN GENERAL;}

With SPECIAL ReFERENCE TO THE “V" WAVE OF ThE POLYGRaphic Tracing.

BY

HARRINGTON SAINSBURY, M.D., F.R.C.P., CONSULTING PEYSIOIAN TO THE ROYAL FREE HOSPITAL AND THE VICTORIA PARK CHEST HOSPITAL.

Is the first place it must be stated that the polygraphio tracing is a fact; it is Nature's own record. This is true, but the statement needs qualification, for an instrument inter. venes between Nature and the recording surface. Nature, therefore, actuates a mechanism, and we must ask, Does the latter transmit faithfully, does it render an exact transcript? This is a primary question, and it raises an element of doubt as to the record.

Passing from the objective side of things to the subjective, we find the observer-another instrument, but one much more complex -intervening between the tracing and its real significance. The observer reads, conjectures, draws conclusions, and makes pronouncement, the judgement speaking the final word. But the judgement, Hippocrates tells us, is difficult, "judicium difficile" (Book I, Aphorism 1), and it is fallible we know. A gain, therefore; there enters an element of doubt, this time as to the interpretation.

Proceeding now to the subject before us let us endeavour to follow, as closely as we are able, the course of the cardiovascular activities, as they repeat themselves in each cardiac cycle; then let us look at the tracings obtained by the poly. graph and see in how far the two harmonize the one with the other.

We shall first note that normally the venous flow from the capillaries on wards presents a smooth current without trace of pulsation. until we come within a certain radius of the 